\title{
Die intramurale Gallengangsvarikose („pseudo-cholangiocarcinoma sign“) in der Computertomographie
}

Die intramurale Gallengangsvarikose ist eine zwar schon beschriebene, jedoch relativ unbekannte Differenzialdiagnose der Gallenwegswandverdickung und muss insbesondere gegenüber einem Gallenwegskarzinom abgegrenzt werden. Wegen dieser Verwechslungsmöglichkeit wurde im angloamerikanischen Sprachraum der Begriff „pseudo-cholangiocarcinoma sign" vorgeschlagen.

\section{Fallbeschreibung}

Bei der 54-jährigen Patientin war seit Jahren eine Pfortaderthrombose mit portaler Hypertension bekannt. Bedingt war die Thrombose wahrscheinlich durch eine chronische Pankreatitis; unklar war, wie lange die Thrombose bereits bestand. Die Patientin stellte sich jetzt vor, um abzuklären, inwieweit eine Shuntanlage möglich sei.

Anamnestisch bekannt waren eine deutliche Splenomegalie, Ösophagusvarizen zweiten bis dritten Grades sowie Fun-

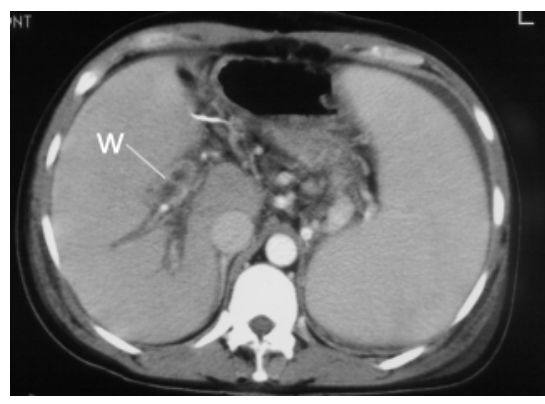

Abb.1 Das computertomographische Bild nach intravenöser Kontrastmittelgabe zeigt eine deutliche Gallenwegswandverdickung (W). Gleichzeitig erkennbare intrahepatische Cholestase sowie Splenomegalie als Zeichen der portalen Hypertension.

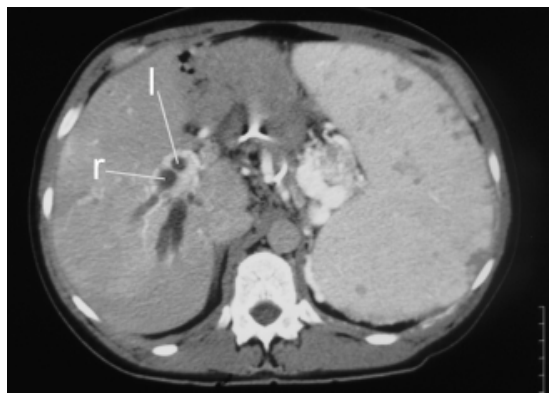

Abb. 2 In der arteriell verstärkten CT (Katheterlage im Truncus coeliacus) zeigt sich eine deutliche Anreicherung dieser Wandverdickung um den rechten ( $r$ ) und linken (I) D. hepaticus.

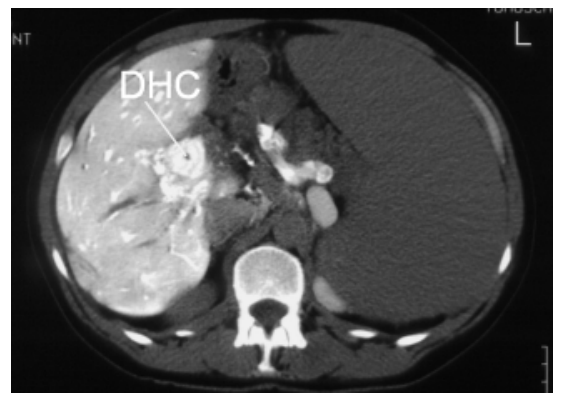

In der durchgeführten computertomographischen Abdomenuntersuchung (Abb.1) zeigte sich die bekannte kavernöse Transformation der Pfortader. Auffallend war eine Erweiterung der intrahepatischen Gallenwege mit deutlicher Wandverdickung des rechten und linken D. hepaticus (Abb. 1,2) sowie des D. hepatocholedochus (Abb.3). Die Wandverdickung betrug bis $\mathrm{zu} 8 \mathrm{~mm}$ und setzte sich als Infiltration bis zum Gallenblasenbett fort, so dass der Verdacht auf ein Gallenwegskarzinom geäußert wurde.

Im Rahmen der weiteren Diagnostik erfolgte eine Angiographie, die den bekannten Pfortaderverschluss mit kaver-
Abb.3 In der portal-venös verstärkten CT (Katheterlage in der A. mesenterica superior) zeigt sich eine noch kräftigere Anreicherung der Gallenwegswandverdickung. Diese setzt sich auf den D. hepatocholedochus (DHC) bzw. in Richtung Gallenblasenbett hin fort. Die extrahepatischen Gallenwege imponieren dabei nicht eingeengt.

nöser Transformation zeigte (Abb.4). Der Verschluss beteiligte auch den Konfluenz und reichte bis auf den konfluenznahen Teil der Milzvene sowie der Vena mesenterica superior. Es fanden sich deutliche Kollateralgefäße im Milzhilus mit Ausbildung eines spontanen spleno- 


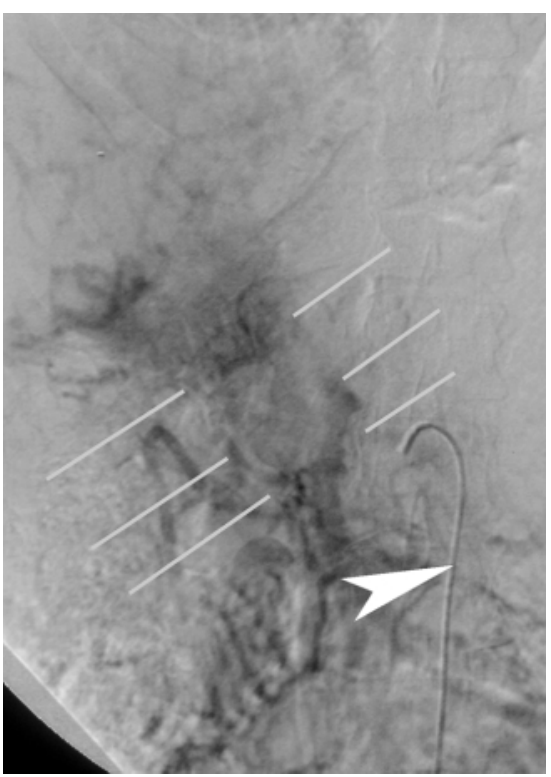

Abb. 4 Venöse Phase einer oberen Mesenterikographie (DSA-Technik). Nach selektiver Kontrastmittelinjektion über einen in der Arteria mesenterica superior platzierten Katheter (Pfeilspitze) findet sich in der venösen Phase der bekannte Pfortaderverschluss mit kavernöser Transformation. Der Verschluss beteiligt auch den Konfluenz und reicht bis auf den konfluenznahen Teil der Milzvene sowie der Vena mesenterica superior. Im Bereich des Ligamentum hepatoduodenale finden sich multiple kleinste Gefäßkonvolute (zwischen den Pfeilen).

renalen Shunts sowie Varizen im Bereich von Magenfundus und distalem Ösophagus.

Im Rahmen der chirurgischen Anlage eines portosystemischen Shunts zwischen V. mesenterica inferior und linker Nierenvene erfolgte auch eine Exploration des Ligamentum hepatoduodenale. Dieses zeigte sich stark verkürzt, verdickt und durchsetzt mit kleinsten, Besenreiservarizen-ähnlichen Gefäßen. Ein maligner Tumor fand sich weder visuell noch palpatorisch. Die Leber war deutlich konsistenzvermehrt und bot makroskopisch die Zeichen einer Fibrose, in einer Keilexzision aus dem Lig. hepatoduodenale fand sich histologisch eine narbenförmige Fibrosierung.

Im Verlauf von jetzt 2 Jahren zeigt sich die Wandverdickung der Gallenwege unverändert.

\section{Diskussion}

Verdickungen der Gallenwegswand finden sich insbesondere beim Gallenwegskarzinom sowie entzündlichen Veränderungen. Die intramurale Gallenwegsvarikose, im angloamerikanischen Sprachraum auch als „pseudo-cholangiocarcinoma sign" bezeichnet, ist eine relativ unbekannte Differenzialdiagnose der Gallenwegswandverdickung. Bedingt ist die Verdickung der Gallenwegswand hierbei durch zahllose kleinste Varizen bei langjährig bestehender kavernöser Pfortadertransformation mit portaler Hypertension (Chow und Jeffrey, AJR 1999; 173 : 1255).

Extensive Kollateralgefäße im Leberhilus können zu einer Kompression und Einengung der Gallenwege, im Einzelfall sogar zu einer Cholestase mit Aufweitung der intrahepatischen Gallenwege führen (Bayraktar et al., Am J Gastroenterol 1992; 87: 1801, Meredith et al., Br J Radiol 1978; 51:1011). Bei unserer Patientin zeigte sich zwar in den bildgebenden Verfahren eine leichte intrahepatische Gallenwegserweiterung, die laborchemischen Parameter einschließlich der alkalischen Phosphatase waren jedoch normal.

Bei den Kollateralvenen im Leberhilus wird zwischen solchen unterschieden, die neben den Gallenwegen, und solchen, die als feines Netzwerk in der Gallenwegswand verlaufen. Größere Varizen bei einer kavernösen Transformation können mittels Farbdopplersonographie, CT oder MRT oft als geschlängelt verlaufende Gefäßkonvolute im Leberhilus (Lig. hepatoduodenale) nachgewiesen werden (Bayraktar et al., Am J Gastroenterol 1992; 87:1801, Kauzlaric et al. MR 1984; 142 : 383, Sugiura et al., J Gastroenterol Hepatol 1993; 8:161). Mittels bildgebender Verfahren kann die eigentliche Pfortader in der Regel nicht mehr dargestellt werden. $\mathrm{Ob}$ es sich bei den Varizen zum Teil um die rekanalisierte Pfortader handelt, wird kontrovers diskutiert. Auch die Abgrenzung dieser Kollateralgefäße gegenüber den extrahepatischen Gallenwegen ist in der alleinigen B-Bildsonographie schwierig, mittels Farbdopplersonographie jedoch in der Regel möglich.
Handelt es sich bei den Kollateralgefäßen um kleinste, in der Gallenwegswand gelegene Gefäße mit einem Durchmesser von weit unter einem Millimeter, kann dies in den bildgebenden Verfahren als Wandverdickung imponieren und so dem Bild eines Gallengangskarzinoms ähneln. Allerdings ist eine längerstreckige, gleichmäßige Wandverdickung für ein Gallenwegskarzinom eher ungewöhnlich.

Auch in der ERCP kann die Gallenwegsvarikose einem Karzinom ähneln. Cholangiographisch wurden Gallenwegsvarizen bei portaler Hypertension zuerst von Williams und Mitarb. (Gastrointest Radiol 1982; 7:69) beschrieben. Im Folgenden wurde über knapp 30 weitere Patienten berichtet. Hauptbefunde waren jeweils eine Einengung sowie noduläre extrinsische Kompression des D. hepaticus communis. Die irreguläre Einengung verlief zum Teil entlang der extrahepatischen Gallenwege und ähnelte einem Gallenwegskarzinom, so dass von Bayraktar und Mitarb. 1992 die Bezeichnung „pseudo-cholangiocarcinoma sign" vorgeschlagen wurde. Bei den meisten der berichteten Patienten lag, wie bei unserer Patientin, eine kavernöse Pfortadertransformation vor im Einzelfall kann eine Gallenwegseinengung durch ausgeprägte Varizen jedoch auch bei offener Pfortader vorkommen (Bayraktar et al., Am J Gastroenterol 1992; 87: 1801).

H. Strunk, J. Textor, J. Remig, Bonn 